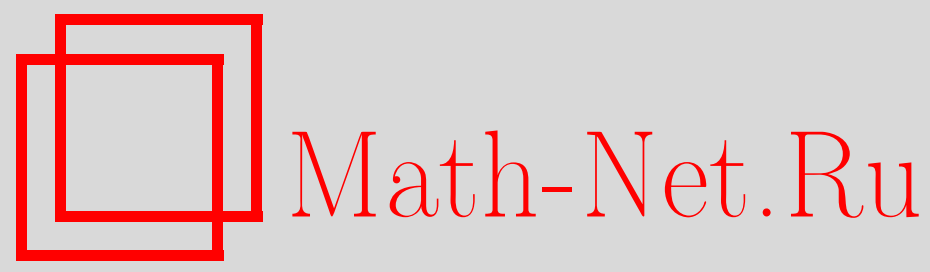

А. Б. Хасанов, А. Б. Яхшимуратов, Некоторые тождества для квадратов компонент собственных вектор-функций системы уравнений Дирака с периодическими коэффициентами, Матем. заметки, 2004, том 76, выпуск 3, 459-465

DOI: https://doi.org/10.4213/mzm112

Использование Общероссийского математического портала Math-Net.Ru подразумевает, что вы прочитали и согласны с пользовательским соглашением http://www . mathnet.ru/rus/agreement

Параметры загрузки:

IP : 3.80 .253 .173

26 апреля 2023 г., 14:33:03

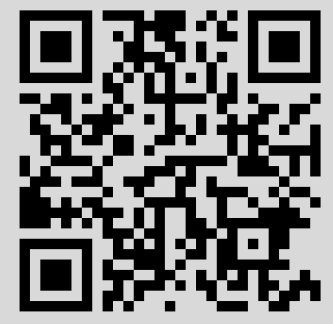




\title{
НЕКОТОРЫЕ ТОЖДЕСТВА ДЛЯ КВАДРАТОВ КОМПОНЕНТ СОБСТВЕННЫХ ВЕКТОР-ФУНКЦИЙ СИСТЕМЫ УРАВНЕНИЙ ДИРАКА С ПЕРИОДИЧЕСКИМИ КОЭФФИЦИЕНТАМИ
}

\author{
А. Б. Хасанов, А.Б. Яхшимуратов
}

В этой работе получены некоторые тождества для квадратов компонент собственных вектор-функций системы уравнений Дирака с гладким периодическим потенциалом.

Библиограффия: 7 названий.

В работах Х. П. Мак-Кина, Е. Трубовица [1] и П. Дейфта, Е. Трубовица [2] получено тождество

$$
\sum_{k=0}^{\infty} \varepsilon_{k} \varphi_{2 k}^{2}(x)=1
$$

для квадратов ортонормированных собственных функций $\varphi_{2 k}(x)$, соответствующим собственньм значениям $\lambda_{2 k}$ оператора Штурма-Лиувилля с периодическими и антипериодическими граничными условиями. Здесь $\varepsilon_{k}$ зависит только от $\lambda_{0}, \lambda_{1}, \lambda_{2}, \ldots$, и является положительньм, если $\lambda_{2 k}-\lambda_{2 k-1}>0$, и равно нулю, если $\lambda_{2 k}-\lambda_{2 k-1}=0$. Это тождество играет важную роль при сведении спектральной задачи для оператора Штурма-Лиувилля с конечнозонным потенциалом к задаче Неймана о движении точки на сфере единичного радиуса под действием квадратичного потенциала (А. П. Веселов [3], Ю. Мозер [4]). Отметим, что подобные тождества имеются и в работах [5], [6].

В настоящей работе получены некоторые тождества для квадратов компонент собственных вектор-функций системы уравнений Дирака с гладким периодическим потенциалом.

Рассмотрим в гильбертовом пространстве вектор-функций $L_{2}^{2}\left(\mathbb{R}^{1}\right)$ оператор Дирака

$$
L y \equiv\left(\begin{array}{cc}
0 & 1 \\
-1 & 0
\end{array}\right)\left(\begin{array}{c}
y_{1}^{\prime} \\
y_{2}^{\prime}
\end{array}\right)+\left(\begin{array}{cc}
p(x) & q(x) \\
q(x) & -p(x)
\end{array}\right)\left(\begin{array}{l}
y_{1} \\
y_{2}
\end{array}\right)=\lambda\left(\begin{array}{l}
y_{1} \\
y_{2}
\end{array}\right), \quad x \in \mathbb{R}^{1}
$$

где $p(x), q(x)$ - действительные, гладкие функции на всей прямой с периодом $\pi$.

Введем решения $\theta(x, \lambda), \varphi(x, \lambda)$ системы $(1)$, удовлетворяющие начальньм условиям $\theta(0, \lambda)=(1,0)^{T}, \varphi(0, \lambda)=(0,1)^{T}$.

Спектр оператора $L$ получается выкидыванием из всей прямой не более, чем счетного числа ограниченных непересекающихся интервалов (называемых лакунами $\left(\lambda_{2 n-1}\right.$, 
$\left.\lambda_{2 n}\right), n \in \mathbb{Z}$. Концы лакун являются собственными значениями либо периодической, либо антипериодической задачи для системы $(1)$ на отрезке $[0, \pi]$ и состоят из нулей целой функции $\Delta^{2}(\lambda)-4$, где $\Delta(\lambda)=\theta_{1}(\pi, \lambda)+\varphi_{2}(\pi, \lambda)$. Кроме того, вьполняется следуюшая асимптотика (см. [7]):

$$
\lambda_{2 n-1}, \lambda_{2 n}=n+\frac{c_{1}}{n}+O\left(\frac{1}{n^{2}}\right), \quad|n| \rightarrow \infty .
$$

Пусть

$$
\ldots, y_{-2}(x), y_{-1}(x), y_{0}(x), y_{1}(x), y_{2}(x), \ldots
$$

- нормированные собственные вектор-функции (периодической и антипериодической задачи для системы (1) на отрезке $[0, \pi])$, соответствуюшие собственным значениям

$$
\cdots \leqslant \lambda_{-2}<\lambda_{-1} \leqslant \lambda_{0}<\lambda_{1} \leqslant \lambda_{2}<\cdots
$$

Основной результат этой работы содержится в следующей теореме.

ТЕОРемА. Для функиий (2) выполняются следующие тождества:

$$
\begin{aligned}
\sum_{k=-\infty}^{\infty} a_{k} y_{2 k-1,1}^{2}(t) & =-p(t)+\frac{c}{2}, & \sum_{k=-\infty}^{\infty} b_{k} y_{2 k, 1}^{2}(t) & =p(t)+\frac{c}{2}, \\
\sum_{k=-\infty}^{\infty} a_{k} y_{2 k-1,2}^{2}(t) & =p(t)+\frac{c}{2}, & \sum_{k=-\infty}^{\infty} b_{k} y_{2 k, 2}^{2}(t) & =-p(t)+\frac{c}{2}, \\
\sum_{k=-\infty}^{\infty} a_{k} y_{2 k-1,1}(t) y_{2 k-1,2}(t) & =q(t), & \sum_{k=-\infty}^{\infty} b_{k} y_{2 k, 1}(t) y_{2 k, 2}(t) & =-q(t),
\end{aligned}
$$

əде

$$
\begin{gathered}
a_{k}=\frac{-\Delta^{\prime}\left(\lambda_{2 k-1}\right)}{f^{\prime}\left(\lambda_{2 k-1}\right)}, \quad b_{k}=\frac{\Delta^{\prime}\left(\lambda_{2 k}\right)}{g^{\prime}\left(\lambda_{2 k}\right)}, \quad c=\sum_{k=-\infty}^{\infty}\left(\lambda_{2 k}-\lambda_{2 k-1}\right), \\
f(\lambda)=\pi\left(\lambda-\lambda_{-1}\right) \prod_{k=1}^{\infty} \frac{\lambda-\lambda_{2 k-1}}{k} \cdot \frac{\lambda-\lambda_{-2 k-1}}{-k}, \\
g(\lambda)=\pi\left(\lambda-\lambda_{0}\right) \prod_{k=1}^{\infty} \frac{\lambda-\lambda_{2 k}}{k} \cdot \frac{\lambda-\lambda_{-2 k}}{-k} .
\end{gathered}
$$

ЗАмечАниЕ. Здесь и в дальнейшем в произведениях $\prod_{k=-\infty}^{\infty}$ и в суммах $\sum_{k=-\infty}^{\infty}$ частичные произведения и частичные суммы берутся симметричным образом.

ДокАЗАТЕЛЬСтво тЕоРемЫ. Введем решения $\theta(x, \lambda, t), \varphi(x, \lambda, t)$ следующей системы уравнений:

$$
\left(\begin{array}{cc}
0 & 1 \\
-1 & 0
\end{array}\right)\left(\begin{array}{l}
y_{1}^{\prime} \\
y_{2}^{\prime}
\end{array}\right)+\left(\begin{array}{cc}
p(x+t) & q(x+t) \\
q(x+t) & -p(x+t)
\end{array}\right)\left(\begin{array}{l}
y_{1} \\
y_{2}
\end{array}\right)=\lambda\left(\begin{array}{l}
y_{1} \\
y_{2}
\end{array}\right), \quad x \in \mathbb{R}^{1}
$$


удовлетворяющие начальным условиям $\theta(0, \lambda, t)=(1,0)^{T}, \varphi(0, \lambda, t)=(0,1)^{T}$, где $t-$ действительньй параметр.

Изучим при $|\lambda| \rightarrow \infty$ асимптотику следующих функций:

$$
\begin{array}{lll}
\frac{\varphi_{1}(\pi, \lambda, t)}{f(\lambda)}, & \frac{-\theta_{2}(\pi, \lambda, t)}{f(\lambda)}, & \frac{\theta_{1}(\pi, \lambda, t)-\varphi_{2}(\pi, \lambda, t)}{f(\lambda)} \\
\frac{\varphi_{1}(\pi, \lambda, t)}{g(\lambda)}, & \frac{-\theta_{2}(\pi, \lambda, t)}{g(\lambda)}, & \frac{\theta_{1}(\pi, \lambda, t)-\varphi_{2}(\pi, \lambda, t)}{g(\lambda)}
\end{array}
$$

Используя разложение

$$
\varphi_{1}(\pi, \lambda, t)=\pi\left(\lambda-\zeta_{0}(t)\right) \prod_{\substack{k=-\infty \\ k \neq 0}}^{\infty} \frac{\lambda-\zeta_{k}(t)}{k},
$$

получим

$$
\begin{aligned}
& \frac{\varphi_{1}(\pi, \lambda, t)}{f(\lambda)}=\sqrt{\prod_{k=-\infty}^{\infty} \frac{\lambda-\lambda_{2 k}}{\lambda-\lambda_{2 k-1}} \cdot \sqrt{\prod_{k=-\infty}^{\infty} \frac{\lambda-\zeta_{k}(t)}{\lambda-\lambda_{2 k-1}} \cdot \frac{\lambda-\zeta_{k}(t)}{\lambda-\lambda_{2 k}}}} \\
& =\sqrt{\exp \left\{\sum_{k=-\infty}^{\infty} \ln \left(1+\frac{\lambda_{2 k-1}-\lambda_{2 k}}{\lambda-\lambda_{2 k-1}}\right)\right\}} \\
& \times \sqrt{\exp \left\{\sum_{k=-\infty}^{\infty} \ln \left[1+\frac{\lambda_{2 k-1}-\xi_{k}(t)}{\lambda-\lambda_{2 k-1}}+\frac{\lambda_{2 k}-\xi_{k}(t)}{\lambda-\lambda_{2 k}}+\frac{\left(\lambda_{2 k-1}-\xi_{k}(t)\left(\lambda_{2 k}-\xi_{k}(t)\right)\right.}{\left(\lambda-\lambda_{2 k-1}\right)\left(\lambda-\lambda_{2 k}\right)}\right]\right\}} \\
& =\sqrt{\exp \left\{\frac{1}{\lambda} \sum_{k=-\infty}^{\infty}\left(\lambda_{2 k-1}-\lambda_{2 k}\right)+O\left(\frac{1}{\lambda^{2}}\right)\right\}} \\
& \times \sqrt{\exp \left\{\frac{1}{\lambda} \sum_{k=-\infty}^{\infty}\left(\lambda_{2 k-1}+\lambda_{2 k}-2 \xi_{k}(t)\right)+O\left(\frac{1}{\lambda^{2}}\right)\right\}}, \quad|\lambda| \rightarrow \infty .
\end{aligned}
$$

Принимая во внимание обозначения (3) и формулу следов

$$
\sum_{k=-\infty}^{\infty}\left(\frac{\lambda_{2 k-1}+\lambda_{2 k}}{2}-\xi_{k}(t)\right)=p(t)
$$

из (4) находим, что

$$
\frac{\varphi_{1}(\pi, \lambda, t)}{f(\lambda)}=1+\frac{1}{\lambda}\left(p(t)-\frac{c}{2}\right)+O\left(\frac{1}{\lambda^{2}}\right), \quad|\lambda| \rightarrow \infty .
$$

На основании равенства

$$
\theta_{1}(\pi, \lambda, t)-\varphi_{2}(\pi, \lambda, t)=\varphi_{1}(\pi, \lambda, t) \sum_{k=-\infty}^{\infty} \frac{\sigma_{k}(t) \sqrt{\Delta^{2}\left(\xi_{k}(t)\right)-4}}{\left(\left.\frac{\partial \varphi_{1}(\pi, \lambda, t)}{\partial \lambda}\right|_{\lambda=\xi_{k}}\right) \cdot\left(\lambda-\xi_{k}(t)\right)}
$$


где $\sigma_{k}(t)=\operatorname{sign}\left\{\theta_{1}\left(\pi, \xi_{k}(t), t\right)-\varphi_{2}\left(\pi, \xi_{k}(t), t\right)\right\}$, и формулы следов

$$
\sum_{k=-\infty}^{\infty} \frac{\sigma_{k}(t) \sqrt{\Delta^{2}\left(\xi_{k}(t)\right)-4}}{\left.\frac{\partial \varphi_{1}(\pi, \lambda, t)}{\partial \lambda}\right|_{\lambda=\xi_{k}}}=q(t)
$$

учитьвая асимптотику (5), вьводим

$$
\frac{\theta_{1}(\pi, \lambda, t)-\varphi_{2}(\pi, \lambda, t)}{f(\lambda)}=\frac{1}{\lambda} q(t)+O\left(\frac{1}{\lambda^{2}}\right), \quad|\lambda| \rightarrow \infty .
$$

Аналогично получаются следующие асимптотики:

$$
\begin{array}{rlrl}
\frac{-\theta_{2}(\pi, \lambda, t)}{f(\lambda)} & =1+\frac{1}{\lambda}\left(-p(t)-\frac{c}{2}\right)+O\left(\frac{1}{\lambda^{2}}\right), & & |\lambda| \rightarrow \infty, \\
\frac{\varphi_{1}(\pi, \lambda, t)}{g(\lambda)}=1+\frac{1}{\lambda}\left(p(t)+\frac{c}{2}\right)+O\left(\frac{1}{\lambda^{2}}\right), & & |\lambda| \rightarrow \infty, \\
\frac{\theta_{1}(\pi, \lambda, t)-\varphi_{2}(\pi, \lambda, t)}{g(\lambda)}=\frac{1}{\lambda} q(t)+O\left(\frac{1}{\lambda^{2}}\right), & & |\lambda| \rightarrow \infty, \\
\frac{-\theta_{2}(\pi, \lambda, t)}{g(\lambda)}=1+\frac{1}{\lambda}\left(-p(t)+\frac{c}{2}\right)+O\left(\frac{1}{\lambda^{2}}\right), & & |\lambda| \rightarrow \infty .
\end{array}
$$

Используя полученные асимптотики и теорему Миттаг-Леффлера, вьводим следующие тождества:

$$
\begin{aligned}
\frac{\varphi_{1}(\pi, \lambda, t)}{f(\lambda)} & =1+\sum_{k=-\infty}^{\infty} \frac{\varphi_{1}\left(\pi, \lambda_{2 k-1}, t\right)}{f^{\prime}\left(\lambda_{2 k-1}\right)} \cdot \frac{1}{\lambda-\lambda_{2 k-1}} \\
\frac{-\theta_{2}(\pi, \lambda, t)}{f(\lambda)} & =1+\sum_{k=-\infty}^{\infty} \frac{-\theta_{2}\left(\pi, \lambda_{2 k-1}, t\right)}{f^{\prime}\left(\lambda_{2 k-1}\right)} \cdot \frac{1}{\lambda-\lambda_{2 k-1}} \\
\frac{\theta_{1}(\pi, \lambda, t)-\varphi_{2}(\pi, \lambda, t)}{f(\lambda)} & =\sum_{k=-\infty}^{\infty} \frac{\theta_{1}\left(\pi, \lambda_{2 k-1}, t\right)-\varphi_{2}\left(\pi, \lambda_{2 k-1}, t\right)}{f^{\prime}\left(\lambda_{2 k-1}\right)} \cdot \frac{1}{\lambda-\lambda_{2 k-1}} \\
\frac{\varphi_{1}(\pi, \lambda, t)}{g(\lambda)} & =1+\sum_{k=-\infty}^{\infty} \frac{\varphi_{1}\left(\pi, \lambda_{2 k}, t\right)}{g^{\prime}\left(\lambda_{2 k}\right)} \cdot \frac{1}{\lambda-\lambda_{2 k}}, \\
\frac{-\theta_{2}(\pi, \lambda, t)}{g(\lambda)} & =1+\sum_{k=-\infty}^{\infty} \frac{-\theta_{2}\left(\pi, \lambda_{2 k}, t\right)}{g^{\prime}\left(\lambda_{2 k}\right)} \cdot \frac{1}{\lambda-\lambda_{2 k}}, \\
\frac{\theta_{1}(\pi, \lambda, t)-\varphi_{2}(\pi, \lambda, t)}{g(\lambda)} & =\sum_{k=-\infty}^{\infty} \frac{\theta_{1}\left(\pi, \lambda_{2 k}, t\right)-\varphi_{2}\left(\pi, \lambda_{2 k}, t\right)}{g^{\prime}\left(\lambda_{2 k}\right)} \cdot \frac{1}{\lambda-\lambda_{2 k}} .
\end{aligned}
$$


Приравнивая коэффициенты при $1 / \lambda$ в равенствах (5)-(8), получим следующие тождества:

$$
\begin{gathered}
\sum_{k=-\infty}^{\infty} \frac{\varphi_{1}\left(\pi, \lambda_{2 k-1}, t\right)}{f^{\prime}\left(\lambda_{2 k-1}\right)}=p(t)-\frac{c}{2}, \quad \sum_{k=-\infty}^{\infty} \frac{-\theta_{2}\left(\pi, \lambda_{2 k-1}, t\right)}{f^{\prime}\left(\lambda_{2 k-1}\right)}=q(t), \\
\sum_{k=-\infty}^{\infty} \frac{\theta_{1}\left(\pi, \lambda_{2 k-1}, t\right)-\varphi_{2}\left(\pi, \lambda_{2 k-1}, t\right)}{f^{\prime}\left(\lambda_{2 k-1}\right)}=-p(t)-\frac{c}{2} \\
\sum_{k=-\infty}^{\infty} \frac{\varphi_{1}\left(\pi, \lambda_{2 k}, t\right)}{g^{\prime}\left(\lambda_{2 k}\right)}=p(t)+\frac{c}{2}, \quad \sum_{k=-\infty}^{\infty} \frac{-\theta_{2}\left(\pi, \lambda_{2 k}, t\right)}{g^{\prime}\left(\lambda_{2 k}\right)}=q(t), \\
\sum_{k=-\infty}^{\infty} \frac{\theta_{1}\left(\pi, \lambda_{2 k}, t\right)-\varphi_{2}\left(\pi, \lambda_{2 k}, t\right)}{g^{\prime}\left(\lambda_{2 k}\right)}=-p(t)+\frac{c}{2}
\end{gathered}
$$

Из равенств (9) и следующей леммы выводим утверждение теоремы.

ЛЕмма. Выполняются следующие равенства:

$$
\begin{gathered}
\varphi_{1}\left(\pi, \lambda_{n}, t\right)=\Delta^{\prime}\left(\lambda_{n}\right) y_{n, 1}^{2}(t), \quad \theta_{2}\left(\pi, \lambda_{n}, t\right)=-\Delta^{\prime}\left(\lambda_{n}\right) y_{n, 2}^{2}(t) \\
\theta_{1}\left(\pi, \lambda_{n}, t\right)=\frac{1}{2} \Delta\left(\lambda_{n}\right)-\Delta^{\prime}\left(\lambda_{n}\right) y_{n, 1}(t) y_{n, 2}(t) \\
\varphi_{2}\left(\pi, \lambda_{n}, t\right)=\frac{1}{2} \Delta\left(\lambda_{n}\right)+\Delta^{\prime}\left(\lambda_{n}\right) y_{n, 1}(t) y_{n, 2}(t)
\end{gathered}
$$

ДоказАтЕЛьСтво. Докажем первое из равенств (10). Случай, когда $\lambda_{n}$ двукратен очевиден. Будем считать, что $\lambda_{n}$ не является двукратным. Из равенств

$$
\begin{aligned}
\theta(t+\pi, \lambda) & =\theta_{1}(\pi, \lambda) \theta(t, \lambda)+\theta_{2}(\pi, \lambda) \varphi(t, \lambda) \\
\varphi(t+\pi, \lambda) & =\varphi_{1}(\pi, \lambda) \theta(t, \lambda)+\varphi_{2}(\pi, \lambda) \varphi(t, \lambda) \\
\varphi(x, \lambda, t) & =\theta_{1}(\pi, \lambda) \varphi(x+t, \lambda)-\varphi_{1}(t, \lambda) \theta(x+t, \lambda)
\end{aligned}
$$

выводим тождество

$$
\varphi_{1}(\pi, \lambda, t)=\varphi_{1}(\pi, \lambda) \theta_{1}^{2}(t, \lambda)+\left[\varphi_{2}(\pi, \lambda)-\theta_{1}(\pi, \lambda)\right] \theta_{1}(t, \lambda) \varphi_{1}(t, \lambda)-\theta_{2}(\pi, \lambda) \varphi_{1}^{2}(t, \lambda)
$$

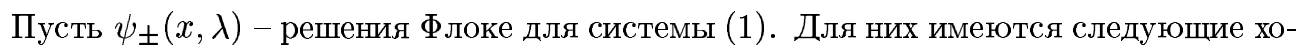
рошо известные представления:

$$
\psi_{ \pm}(x, \lambda)=\theta(x, \lambda)+\frac{\varphi_{2}(\pi, \lambda)-\theta_{1}(\pi, \lambda) \mp \sqrt{\Delta^{2}(\lambda)-4}}{2 \varphi_{1}(\pi, \lambda)} \varphi(x, \lambda) .
$$

Введем обозначение

$$
\psi\left(x, \lambda_{n}\right)=\psi_{ \pm}\left(x, \lambda_{n}\right)=\theta\left(x, \lambda_{n}\right)+\frac{\varphi_{2}\left(\pi, \lambda_{n}\right)-\theta_{1}\left(\pi, \lambda_{n}\right)}{2 \varphi_{1}\left(\pi, \lambda_{n}\right)} \varphi\left(x, \lambda_{n}\right) .
$$


Из формулы

$$
\begin{aligned}
& \Delta^{\prime}(\lambda)=\varphi_{1}(\pi, \lambda) \int_{0}^{\pi}\{ {\left[\theta_{1}(x, \lambda)+\frac{\varphi_{2}(\pi, \lambda)-\theta_{1}(\pi, \lambda)}{2 \varphi_{1}(\pi, \lambda)} \varphi_{1}(t, \lambda)\right]^{2} } \\
&\left.+\left[\theta_{2}(x, \lambda)+\frac{\varphi_{2}(\pi, \lambda)-\theta_{1}(\pi, \lambda)}{2 \varphi_{1}(\pi, \lambda)} \varphi_{2}(t, \lambda)\right]^{2}\right\} d t \\
&-\frac{\Delta^{2}(\lambda)-4}{4 \varphi_{1}(\pi, \lambda)} \int_{0}^{\pi}\left[\varphi_{1}(t, \lambda)+\varphi_{2}^{2}(t, \lambda)\right] d t
\end{aligned}
$$

находим

$$
\Delta^{\prime}\left(\lambda_{n}\right)=\varphi_{1}\left(\pi, \lambda_{n}\right) \int_{0}^{\pi}\left[\psi_{1}^{2}\left(t, \lambda_{n}\right)+\psi_{2}^{2}\left(t, \lambda_{n}\right)\right] d t
$$

На основании простоты собственного значения $\lambda_{n}$ выводим равенство

$$
\psi\left(t, \lambda_{n}\right)= \pm \sqrt{\frac{\Delta^{\prime}\left(\lambda_{n}\right)}{\varphi_{1}\left(\pi, \lambda_{n}\right)}} \cdot y_{n}(t)
$$

Отсюда имеем

$$
\varphi_{1}\left(\pi, \lambda_{n}\right) \psi_{1}^{2}\left(t, \lambda_{n}\right)=\Delta^{\prime}\left(\lambda_{n}\right) y_{n, 1}^{2}(t)
$$

Используя (11), (12) и тождество

$$
\left[\theta_{1}(\pi, \lambda)-\varphi_{2}(\pi, \lambda)\right]^{2}=\Delta^{2}(\lambda)-4-4 \varphi_{1}(\pi, \lambda) \theta_{2}(\pi, \lambda)
$$

преобразуем левую часть равенства (13):

$$
\begin{aligned}
\varphi_{1}( & \left.\pi, \lambda_{n}\right) \psi_{1}^{2}\left(t, \lambda_{n}\right) \\
= & \frac{1}{4 \varphi_{1}\left(\pi, \lambda_{n}\right)}\left\{2 \varphi_{1}\left(\pi, \lambda_{n}\right) \theta_{1}\left(t, \lambda_{n}\right)+\left[\varphi_{2}\left(\pi, \lambda_{n}\right)-\theta_{1}\left(\pi, \lambda_{n}\right)\right] \varphi_{1}\left(t, \lambda_{n}\right)\right\}^{2} \\
= & \frac{1}{4 \varphi_{1}\left(\pi, \lambda_{n}\right)}\left\{4 \varphi_{1}^{2}\left(\pi, \lambda_{n}\right) \theta_{1}^{2}\left(t, \lambda_{n}\right)\right. \\
& +4 \varphi_{1}\left(\pi, \lambda_{n}\right)\left[\varphi_{2}\left(\pi, \lambda_{n}\right)-\theta_{1}\left(\pi, \lambda_{n}\right)\right] \cdot \theta_{1}\left(t, \lambda_{n}\right) \varphi_{1}\left(t, \lambda_{n}\right) \\
& \left.-4 \varphi_{1}\left(\pi, \lambda_{n}\right) \theta_{2}\left(\pi, \lambda_{n}\right) \varphi_{1}^{2}\left(t, \lambda_{n}\right)\right\} \\
= & \varphi_{1}\left(\pi, \lambda_{n}\right) \theta_{1}^{2}\left(t, \lambda_{n}\right)+\left[\varphi_{2}\left(\pi, \lambda_{n}\right)-\theta_{1}\left(\pi, \lambda_{n}\right)\right] \theta_{1}\left(t, \lambda_{n}\right) \varphi_{1}\left(t, \lambda_{n}\right)-\theta_{2}\left(\pi, \lambda_{n}\right) \varphi_{1}^{2}\left(t, \lambda_{n}\right) \\
= & \varphi_{1}\left(\pi, \lambda_{n}, t\right) .
\end{aligned}
$$

Учитьвая (13), вьводим первое тождество леммы; остальные равенства доказьваются аналогично. Лемма доказана.

Отметим, что в случаях $p(x) \equiv q(x) \equiv 0$ и $p(x) \equiv m, m>0, q(x) \equiv 0$ полученные тождества принимают вид числовых равенств. 


\section{СПИСОК ЦИТИРОВАННОЙ ЛИТЕРАТУРЫ}

[1] McKean H. P., Trubowitz E. Hill's operator and hyperelliptic function theory in the presence of infinitely many branch points // Comm. Pure Appl. Math. 1976. V. 29. P. 143-226.

[2] Deift P., Trubowitz E. An identity among squares of eigenfunctions // Comm. Pure Appl. Math. 1981. V. 34. P. 713-717.

[3] Веселов А. П. Конечнозонные потенциалы и интегрируемые системы на сфере с квадратичньмм потенциалом // Функцион. анализ и его прилож. 1980. Т. 14. №1. С. 48-50.

[4] Мозер Ю. Некоторые аспекты интегрируемых гамильтоновых систем // УМН. 1981. Т. 36. № 5 (221). C. 109-151.

[5] Савин А. В. Тождество для квадратов собственных функций бесконечнозонного оператора Штурма-Лиувилля // Вестн. Моск. ун-та. Сер. 1. Матем. мех. 1984. №6. С. 83-85.

[6] Хасанов А. Б., Ибрагимов А. М. Об обратной задаче для оператора Дирака с периодическим потенциалом // Узб. матем. ж. 2001. № 3-4. С. 48-55.

[7] Левитан Б.М., Саргсян И.С. Операторы Штурма-Лиувилля и Дирака. М.: Наука, 1988.

Ургенчский государственный университет, Узбекистан

Поступило

E-mail: ahasanov2002@mail.ru 23.05 .2003 